\title{
Observing the Epoch of Reionization with the Cosmic Microwave Background
}

\author{
Christian L. Reichardt
}

\section{Introduction}

Our earliest view of the Universe comes from the cosmic microwave background (CMB), and as such, observations of the CMB have proven to be an invaluable tool in modern cosmology. $\mathrm{CMB}$ experiments will continue to play a vital role in testing the standard cosmological model in the future, for instance in studying inflation and neutrino physics. With this motivation, CMB experiments have rapidly advanced following the first detection of temperature anisotropy using the DMR experiment on the COBE satellite (Smoot et al. 1992), and experimental sensitivities continue to improve today. Experiments being built right now have an order of magnitude more detectors than ever before, and experiments are being proposed with more than a hundred times as many detectors (Abazajian et al. 2014). A key distinction between the $\mathrm{CMB}$ and other proposed probes of reionization such as $21 \mathrm{~cm}$ surveys, is that the CMB is a 2-dimensional measurement with the observables integrated along the line of sight; this rules out techniques like redshift tomography. Despite their 2D nature, $\mathrm{CMB}$ measurements have yielded two significant constraints on cosmic reionization to date.

The first major constraint on cosmic reionization came with the detection of the so-called "reionization" bump in large-scale CMB polarization by the WMAP satellite Kogut et al. (2003); the uncertainty on this measurement has decreased with each successive WMAP data release. Thomson scattering between the free electrons released by cosmic reionization and the local CMB quadrupole produces linear polarization at the horizon scale during the epoch of reionization (EoR). The scattered power depends on the square of the optical depth $\tau$, so the reionization bump in polarization can break a degeneracy between $\tau$ and the amplitude of the primordial scalar perturbations $A_{S}$ that exists in the temperature anisotropy alone. The optical

Christian L. Reichardt

School of Physics, University of Melbourne, Parkville, VIC 3010, Australia, e-mail: christian.reichardteunimelb.edu.au 
depth and scalar amplitude are otherwise degenerate because, for example, observing less CMB anisotropy could be explained either by increasing $\tau$ or reducing $A_{s}$. Galactic foregrounds pose a significant challenge in measuring polarization on these 10 s of degree angular scales, and multiple frequencies are essential to disentangling the signals. Using measurements of the $\ell<20 \mathrm{TE}$ and $\mathrm{EE}^{1}$ power spectra from the WMAP satellite, Bennett et al. (2013) find $\tau=0.089 \pm 0.014$. Data from the Planck satellite (and potentially other future experiments) are expected to improve upon this measurement, with the fundamental cosmic variance limit being lower by roughly a factor of seven. The optical depth depends on the electron number density integrated along the line of sight, and thus depends primarily on when the Universe reionizes.

The second major CMB constraint on cosmic reionization comes from upper limits on the kinematic Sunyaev-Zel'dovich (kSZ) effect. Two sources contribute to the $\mathrm{kSZ}$ power spectrum: density variations in the late-time fully ionized Universe (homogenous $\mathrm{kSZ}$ ) and ionization fraction variations during the EoR (patchy $\mathrm{kSZ}$ ). In the standard picture of reionization, ionized bubbles form around early UV sources, with these bubbles eventually merging to form the completely ionized Universe. The relative velocity between these bubbles and the CMB Doppler shifts the scattered light, which translates to a temperature shift in the CMB along that line of sight. The angular dependence of the kSZ power spectrum depends on the details of these bubbles, which in turn depend on the nature of the ionizing sources and the sinks of ionizing photons (i.e. structure in the intergalactic medium) (Mesinger et al. 2012; Sobacchi \& Mesinger, 2014). The magnitude of the kSZ power from reionization will scale with the number of bubbles, and therefore the duration of the EoR. The ACT and SPT-SZ surveys have published consistent upper limits on the kSZ power Addison et al. (2013); Dunkley et al. (2013); George et al. (2015), with the most stringent published $95 \%$ CL upper limit on the patchy kSZ power being $D_{\mathrm{kSZ}}<3.3 \mu \mathrm{K}^{2}$ at $\ell=3000$ from the $2500 \mathrm{deg}^{2}$ SPT-SZ survey George et al. (2015). These upper limits on the $\mathrm{kSZ}$ power suggest cosmic reionization was not a slow process.

In Section 2, we review the physics of how the optical depth affects the CMB, current measurements, and forecasts for future experiments. We do the same for the $\mathrm{kSZ}$ power spectrum in Section 3 . We review other potential observational consequences of cosmic reionization on the CMB in Section 4, before concluding in Section 5

\section{Mean Optical Depth}

\subsection{Theory}

The transition of the Universe from a neutral to ionized state dramatically increases the number density of free electrons that can Thomson scatter CMB photons. The

\footnotetext{
${ }^{1} \mathrm{~T}$ stands for temperature anisotropy and $\mathrm{E}$ for polarized E-mode (zero curl) anisotropy.
} 


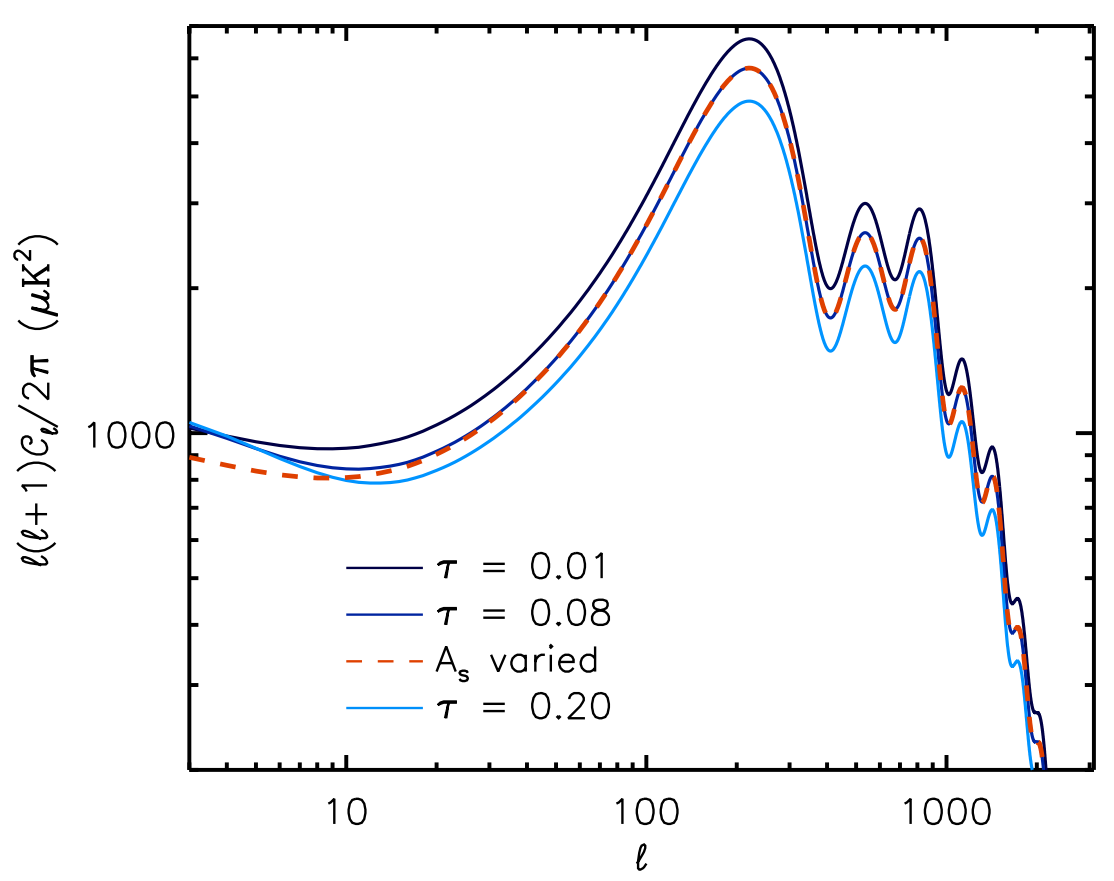

Fig. 1 The optical depth $\tau$ and primordial amplitude of scalar perturbations $A_{s}$ have nearly identical effects on the CMB temperature power spectrum. The impact of increasing optical depth is shown by black to light blue lines: $\tau=0.01,0.08,0.20$. The anisotropy power is reduced by a factor $e^{-2 \tau}$ at all scales smaller than the horizon size at $\operatorname{EoR}(\ell \gtrsim 20)$. This reduction is nearly perfectly degenerate with a shift in the amplitude of the primordial power spectrum. The degeneracy in the temperature power spectrum is illustrated by the dashed red line, which mimics the dark blue $\tau=0.08$ line by reducing the primordial amplitude of scalar perturbations, $A_{s}$.

probability that a given photon will scatter can be related to an effective optical depth to reionization:

$$
\tau=\int n_{e} \sigma_{T} d \ell
$$

Here, $n_{e}$ is the free electron number density, $\sigma_{T}$ is the Thompson cross-section, and the integral is over the line of sight distance. The integral is dominated by electrons from singly-ionized Hydrogen and Helium; doubly ionized Helium at low redshift accounts for a few percent of the total optical depth. Due to its integral nature, the optical depth is insensitive to the precise evolution in the ionization fraction. However, as the median redshift of reionization increases so will the column depth of free electrons and $\tau$. Therefore constraints on $\tau$ are sometimes expressed as constraints on the redshift of reionization, with only weak dependence on the assumed ionization history.

The optical depth suppresses the CMB anisotropy power at all scales smaller than the horizon size at the EoR by a factor $e^{-2 \tau}$. This suppression is shown in 


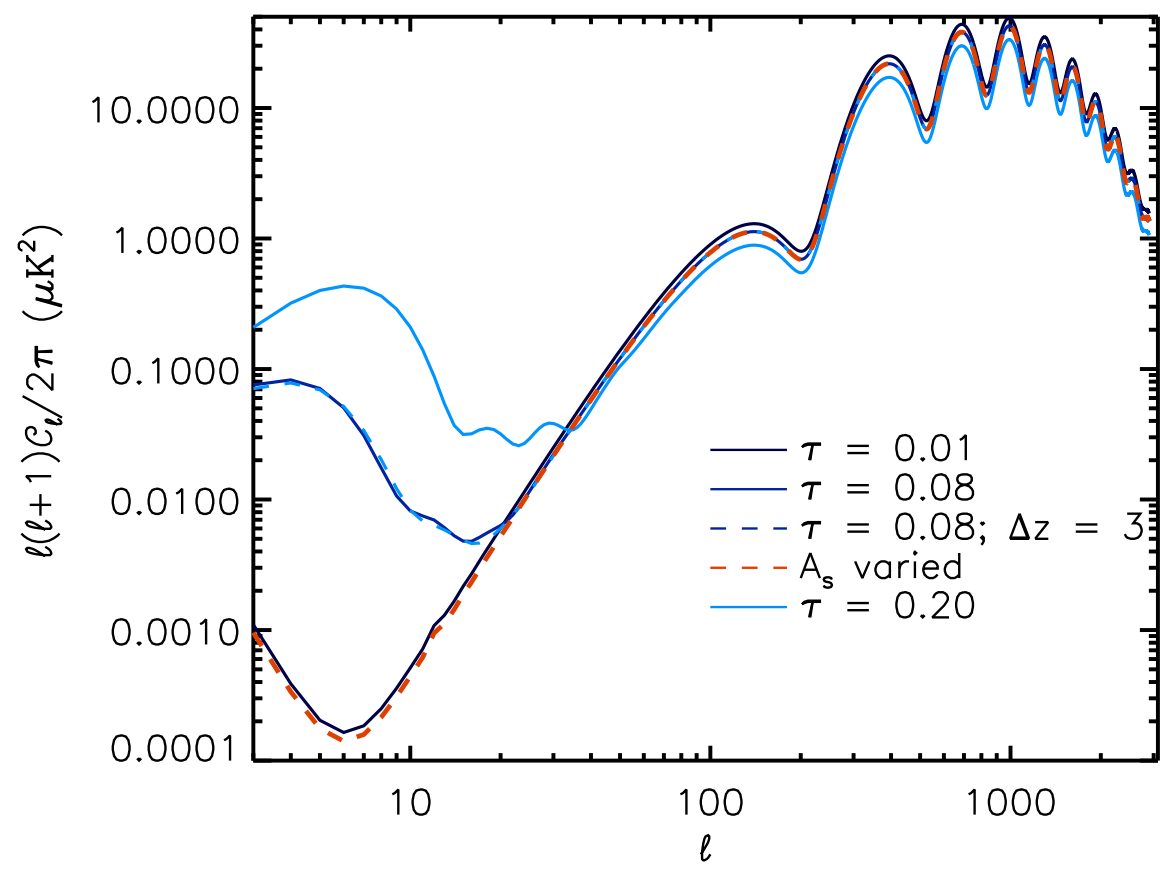

Fig. 2 The impact of optical depth on the CMB $E$-mode (zero curl) polarization power spectrum. The solid lines show the E-mode power spectrum as the optical depth is increased: from black to light blue, $\tau=0.01,0.08,0.20$. E-mode polarization is produced by Thomson scattering between electrons and the CMB quadrupole. As $\tau$ increases, the polarization power spectrum is increased on scales larger than the horizon size at EoR $(\ell \lesssim 20)$. The induced polarized power scales as $\tau^{2}$, and yields the strongest constraint from the $\mathrm{CMB}$ on the optical depth. Additionally as in the temperature anisotropies, the polarization power is reduced by a factor $e^{-2 \tau}$ at all scales larger than the horizon size at $\operatorname{EoR}(\ell \gtrsim 20)$. As in the temperature power spectrum plot, the dashed red line mimics the $\tau=0.08$ line at small scales by reducing the primordial amplitude of scalar perturbations, $A_{s}$ instead of increasing $\tau$. However, decreasing $A_{s}$ does not reproduce the peak at large angular scales (low $\ell$ ). Finally, the dashed blue line has the same optical depth $(\tau=0.08)$ but an EoR duration increased by $6 \times$ to $\Delta z=3$; the $E$-mode power barely changes. The $E$-mode power is largely insensitive to differences between EoR scenarios that produce the same total optical depth.

Figure 1 for $\left.\tau \in[0.01,0.20]\right|^{2}$ Although the magnitude of the suppression is quite large compared to measurement uncertainties, the suppression is highly degenerate with the amplitude, $A_{s}$, of the primordial power spectrum of scalar perturbations. This point is illustrated by the red, dashed line in Figure 1 which reduces $A_{s}$ to mimic the $\tau=0.08$ line. The effect of the two parameters differs only at $\ell \lesssim 20$, and the substantial cosmic variance at these large angular scales prevents a meaningful separation with temperature data alone.

${ }^{2}$ All spectra in this chapter were calculated using CAMB Lewis et al. (2000); Howlett et al. 2012.). 
The reionization of the Universe also creates CMB polarization. In general, scattered radiation from an electron in a quadrupole radiation field will be linearly polarized (see e.g., Rees, 1968). Thus reionization leads to linear polarization as free electrons from reionization are exposed to the large-scale CMB quadrupole. The polarized signal peaks on scales larger than the horizon at EoR; the signal amplitude scales as $\tau$ and thus the power scales as $\tau^{2}$. This signal is sometimes referred to as the reionization "bump" for reasons illustrated in Fig. 22 Importantly, no other parameter in the standard cosmological model produces such a feature so the parameter degeneracies are minimal. For better or worse, the polarized signal encodes very little information beyond the optical depth, as demonstrated by comparing the dashed and solid blue lines in Fig. 2 A cosmic variance limited experiment would measure at most a couple of parameters beyond optical depth Baumann et al.(2009). One downside is that polarized galactic emission is major concern on these large scales (see, e.g., Bennett et al. (2013) for a discussion of foreground modeling). Finally, the number of independent modes on the sky is relatively low, which sets a fundamental cosmic variance limit on how well the amplitude can be measured, although this limit is well below current measurements. The reionization bump is a clean probe of the optical depth to reionization.

\subsection{Current Observations}

The polarization signal from reionization ("reionization bump") was first detected by looking at the temperature-polarization correlation in the first year of data from the WMAP satellite Kogut et al. (2003). The reionization bump in the EE power spectrum was first detected in the 3-year WMAP bandpowers Page et al. (2007). These measurements broke the previous degeneracy between $A_{S}$ and $\tau$ from the temperature data, and substantially improved cosmological constraints. WMAP is still the only experiment to have measured the reionization signal; the history of the published values is tabulated in Table 1 . Essentially, this is due to the large angular scales involved - large scales strongly favor satellite experiments that cover the whole sky. The WMAP measurement is limited by a combination of instrumental noise and uncertainty in the galactic foreground modeling.

The WMAP polarized galactic foreground model includes two terms, dust and synchrotron (see Bennett et al. (2013)) The polarized synchrotron template is taken from the lowest WMAP frequency band (K band at $22 \mathrm{GHz}$ ). The polarized dust template starts from the model 8 dust intensity map from Finkbeiner et al. (1999), with the amplitude modulated by a term to account for the magnetic field geometry, and a polarization direction taken from starlight measurements. The uncertainty due to the foreground modeling are on par with the statistical uncertainties.

Recent results from the Planck satellite suggest that a more accurate galactic dust template reduces the inferred optical depth (Planck Collaboration et al., 2014a. 2015). In Appendix E, Planck Collaboration et al. (2014a) find that replacing the dust template used by WMAP with the Planck $353 \mathrm{GHz}$ map changes $\tau$ by $1 \sigma$ to 
$\tau=0.075 \pm 0.013$. This drop persisted through the second Planck release. Planck Collaboration et al. (2015) found values of $\tau=0.071 \pm 0.013$ when cleaning the large-scale WMAP9 polarization data by a polarized galactic dust template based on Planck's polarized $353 \mathrm{GHz}$ maps. Replacing the WMAP polarization data by Planck $70 \mathrm{GHz}$ polarization data leads to a weaker but consistent constraint of $\tau=0.078 \pm 0.019$. The joint constraint from the WMAP and Planck $70 \mathrm{GHz}$ polarization data is $\tau=0.074 \pm 0.012$. Setting aside all polarization data and instead using only Planck temperature and lensing measurements yields $\tau=0.070 \pm 0.024$. These measurements are all consistent with each other and favor a lower optical depth with a midpoint to reionization around $z \simeq 9$ instead of 10.6. A lower optical depth would reduce the marginal tension between current CMB data and suggestions from the spectra of quasars or gamma ray bursts (e.g., Mortlock et al., 2011, Schroeder et al. 2013), and the dropoff in the luminosity function of Ly $\alpha$ emitters (e.g., Ouchi et al. 2010; Clément et al., 2012) and in the Ly $\alpha$ fraction (e.g., Treu et al. 2013; Caruana et al. 2014) that reionization ended between a redshift of 6 to 7 (see also Figure 4). We will learn more with the release of the full Planck polarization results (see next section).

\begin{tabular}{l|c}
\hline \hline Source & $\tau$ \\
\hline WMAP1 & $0.17 \pm 0.04$ \\
WMAP3 & $0.089 \pm 0.030$ \\
WMAP5 & $0.087 \pm 0.017$ \\
WMAP7 & $0.088 \pm 0.015$ \\
WMAP9 & $0.089 \pm 0.014$ \\
\hline with Planck TT and 353 GHz dust template: & $0.071 \pm 0.013$ \\
WMAP9 pol. & $0.078 \pm 0.019$ \\
Planck LFI pol. & $0.067 \pm 0.022$ \\
Planck LFI pol. (no TT) & $0.067 \pm 0.016$ \\
\hline Planck lensing + BAO
\end{tabular}

Table 1 The optical depth measured by WMAP and Planck across data releases. WMAP's measurement of the optical depth has been relatively static after the second WMAP data release Page et al. (2007). Differences between the first and second data release include: (1) $\tau$ is derived primarily from $E E$ instead of $T E$, (2) three times the data volume, (3) a new foreground treatment, and (4) an updated polarization analysis. An updated polarized galactic dust template based on the $353 \mathrm{GHz}$ channel of Planck/HFI reduces the optical depth by $\sim 1 \sigma$ (bottom half of table). The low- $\ell$ polarization data from $70 \mathrm{GHz}$ in Planck favors an even lower optical depth; a value that is consistent with estimates that avoid using the large-scale polarization data at all. Future releases of the Planck satellite are expected to achieve $\sigma(\tau) \sim 0.005$ and the cosmic variance limit is $\sigma(\tau) \sim 0.002$. 


\subsection{Future Observations}

Optical depth constraints from the full polarization analysis of the Planck satellite are expected to be released towards the end of 2015. Based on sensitivities from the Planck bluebook The Planck Collaboration (2006), the Planck polarized noise should be substantially lower than WMAP. Optical depth forecasts are challenging beause the Planck constraint is limited by the foreground subtraction rather than instrumental noise. However, The Planck Collaboration (2006) predict $1 \sigma$ error bars of $\sigma(\tau) \simeq 0.005$.

A number of proposed satellite experiments hope to improve upon the Planck result and reach the fundamental cosmic variance limit at $\sigma(\tau) \simeq 0.002$. These include CMBpol Baumann et al. (2009), LiteBird Hazumi et al. (2012), and PIXIE Kogut et al. (2011). There are also a handful of ground- or balloon-based experiments, such as GroundBird Tajima et al. (2012), that might be able to measure these large scales.

\section{Kinematic Sunyaev-Zel'dovich effect}

\subsection{Theory and modeling}

The second observational signature of the EoR on the CMB is the kinematic Sunyaev-Zel'dovich (kSZ) effect. The bulk velocity of free electrons relative to the CMB will introduce a Doppler shift to the scattered photons, an effect known of the kSZ effect Sunyaev \& Zel'dovich (1972); Phillips (1995); Birkinshaw (1999); Carlstrom et al. (2002). In the non-relativistic limit, the kSZ effect slightly changes the observed CMB temperature, with the temperature shift scaling as $(v / c) n_{e}$ where $v$ is the line-of-sight bulk velocity of electrons, $c$ is the speed of light and $n_{e}$ is the density of free electrons. The result is a hot spot if the ionized gas is moving towards the observer and a cold spot if moving away.

The total kSZ signal along a line of sight is:

$$
\frac{\Delta T_{\mathrm{kSZ}}}{T_{C M B}}(\hat{\mathbf{n}})=\sigma_{T} \bar{n}_{e, 0} \int d \eta a^{-2} e^{-\tau(\eta)} \bar{x}_{e}(\eta)\left(1+\delta_{x}\right)\left(1+\delta_{\mathrm{b}}\right)(-\hat{\mathbf{n}} \cdot \mathbf{v}),
$$

where $\sigma_{T}$ is Thomson scattering cross-section, $\tau(\eta)$ is the optical depth from the observer to conformal time $\eta, \bar{x}_{e}(\eta)$ is the mean ionization fraction at $\eta, a$ is the scale factor at $\eta$, and $\bar{n}_{e, 0}$ is the mean electron density of the universe today. Perturbations in the baryon density and ionization fraction are marked by $\delta_{\mathrm{b}}$ and $\delta_{\mathrm{x}}$ respectively. Finally, $\hat{\mathbf{n}}$ is the line of sight unit vector and $\mathbf{v}$ represents the peculiar velocity of free electrons at $\eta$. A net $\mathrm{kSZ}$ signal thus requires perturbations in the free electron number density that are correlated with the large-scale velocity field.

The kSZ signal is naturally divided into two components. The homogenous kSZ signal is sourced by perturbations in the density $\left(\delta_{\mathrm{b}}\right)$ of the fully ionized Universe. 
The patchy kSZ signal is sourced by perturbations in the ionization fraction $\left(\delta_{x}\right)$ during reionization. Ionized bubbles are expected to form around the first stars, galaxies, and quasars. These bubbles eventually overlap and merge, leading to a fully ionized universe. The proper motion of an ionized bubble generates angular anisotropy through the kSZ effect. These bubbles are generically correlated with the velocity field because the ionizing sources are biased tracers of the matter distribution. The velocity dependence also means that larger simulation volumes are required to properly estimate the sample variance; the auto-correlation length of the velocity field is order $100 \mathrm{Mpc}$ whereas that of the reionization field is order $10 \mathrm{Mpc}$. The patchy and homogenous kSZ components are expected to have comparable power.

The amplitude of this patchy kSZ power depends primarily on the duration of reionization, while its shape depends on the distribution of bubble sizes. Both features also depend more weakly on the average redshift of reionization Gruzinov \& $\mathrm{Hu}$ (1998); Knox et al. (1998); Santos et al. (2003); Zahn et al. (2005); McQuinn et al. (2005); Iliev et al. (2006); Zahn et al. (2012); Mesinger et al. (2012); Battaglia et al. (2013); Calabrese et al. (2014). The qualitative behavior of these dependencies can understood simply: the power is linearly proportional to the number of bubbles along the line of sight which scales with the duration. Similarly if the bubbles are larger, the kSZ power will peak at larger scales and vice versa. If cosmic reionization occurs at earlier times, the Universe is denser and the same duration leads to more kSZ power.

There are two potentially important qualifications to this picture. First, the signal depends on reionization being inhomogenous. Perfectly homogenous sources can reionize the Universe without producing any $\mathrm{kSZ}$ power. However, in practice, this is unlikely to be significant. Recent work by Mesinger et al. (2013) shows that reionization by ultra-hard $\mathrm{x}$-rays (which have corresponding long mean free paths) reduce the kSZ power by less than $0.5 \mu \mathrm{K}^{2}$. Second, observations typically probe a specific angular scale, and therefore a particular weighting of bubble sizes. Simulations that change the angular shape, whether by changing the mean free path of ionizing photons and thus bubble sizes Mesinger et al. (2012) or suppressing bubbles of the relevant size with a self-regulation mechanism Park et al. (2013), result in a more complicated relationship between the midpoint and duration of EoR than found by Zahn et al. (2012) and others.

Current data can not distinguish between the homogenous and patchy kSZ components because both components have the same spectral dependence and similar angular dependencies. Thus the EoR inferences depend on accurately modeling (and subtracting) the homogenous contribution. The homogenous kSZ power spectrum has been simulated by a number of authors (e.g., Trac et al. 2011; Shaw et al., 2012). Recent predictions for the homogenous kSZ power at $\ell=3000$ range from 2.2 to $3.2 \mu \mathrm{K}^{2}$ for a common cosmology, a peak-peak range of $\pm 20 \%$. This modeling uncertainty is subdominant to current statistical uncertainties, although this is likely to change with upcoming experiments. Future experiments might be able to separate the components using the angular dependence (although this will be challenging), higher-order moments of the map, or cross-correlation with other observables Shaw et al. (2012). 


\subsection{Current Data}

Figure 3 shows current measurements of the kSZ power at $\ell=3000$ using data from the Planck, ACT and SPT-SZ experiments Addison et al. (2013); Dunkley et al. (2013); Planck Collaboration et al. (2014b); George et al. (2015). Using the $2500 \mathrm{deg}^{2}$ SPT-SZ survey, George et al. (2015) report the $\mathrm{kSZ}$ power at $\ell=3000$ to be $D_{\mathrm{kSZ}}=2.9 \pm 1.3 \mu \mathrm{K}^{2}$. The measured power is consistent between experiments. Since the homogenous signal is expected to be above $2.0 \mu \mathrm{K}^{2}$ Shaw et al. (2012), the observed kSZ power leaves little room for patchy kSZ power. George et al. (2015) combine these facts to set an $95 \%$ confidence level upper limit on the patchy $\mathrm{kSZ}$ power at $\ell=3000$ of $D_{\mathrm{kSZ}}^{\text {patchy }}<3.3 \mu \mathrm{K}^{2}$. The measured $\mathrm{kSZ}$ power (from SPT-SZ) and optical depth (from WMAP) is used to importance sample a suite of reionization models as described by Zahn et al. (2012), and infer constraints on the EoR. Defining the duration of reionization as the time the Universe takes to go from a $20 \%$ to 99\% volume-averaged ionization fraction, the patchy $\mathrm{kSZ}$ limit translates to a $95 \%$ confidence level upper limit ${ }^{3}$ on the duration of $\Delta z<5.4$ George et al. (2015).

The power at these few-arcminute angular scales is a combination of the thermal and kinematic SZ effects, radio galaxies and dusty galaxies. In principle these components can be separated cleanly based on each one's unique frequency dependence. However in practice substantial degeneracies remain between the measured thermal and kinematic SZ powers because of the limited frequency coverage. The degeneracy in current data between the two SZ effects can be partially broken by considering higher order moments of the map 4 as is done in George et al. (2015), or by including additional data at other frequencies Addison et al. (2013). Current kSZ measurements are limited by the ability to separate these different signals.

\subsection{Future Observations}

In the near term, there is the potential to combine multi-frequency data from several experiments to better measure the kSZ power. The Planck satellite has multifrequency coverage, but its coarse angular resolution makes it difficult to access the relevant small angular scales. Another recent satellite, HERSCHEL, has made confusion-noise-limited maps of the cosmic infrared background (CIB) across hundreds of square degrees at frequencies $8-16 \times$ higher than the SPT-SZ observing bands. Modulo modeling uncertainties in understanding the CIB over such a wide frequency range, the HERSCHEL data could be used to constrain the CIB and break the degeneracy between the tSZ-CIB correlation and kSZ that limits current mea-

\footnotetext{
${ }^{3}$ This limit does not include modeling uncertainties from reionization scenarios not included in simulation suite used by Zahn et al. (2012).

${ }^{4}$ Higher order moments can break the degeneracy because the degree of non-Gaussianity varies between components.
} 

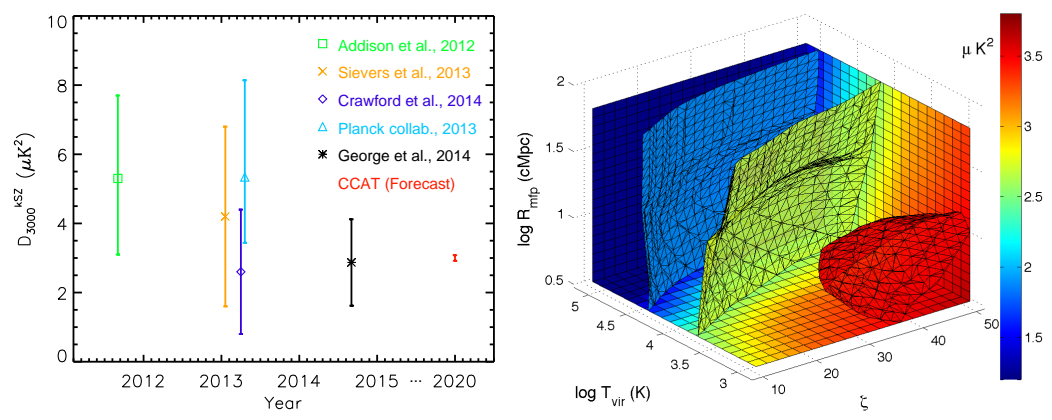

Fig. 3 Left panel: Published $1 \sigma$ constraints on the total kSZ power at $\ell=3000$ ordered by arxiv release date. The most recent two constraints using data from ACT (green square Addison et al. (2013) and orange $x$ Sievers et al. (2013)) and SPT-SZ (purple diamond Crawford et al. (2014) and black asterisk George et al. (2015)) are shown. The Planck result (light blue triangle Planck Collaboration et al. (2014b)) uses both ACT and SPT-SZ data. Also shown in red is a forecast (arbitrarily centered at $3 \mu \mathrm{K}^{2}$ ) for the proposed LWCAM instrument on CCAT which should begin taking data by 2020 . Upcoming experiments like CCAT, AdvACT, and SPT-3G should dramatically improve measurements of the kSZ power spectrum. Right panel: The patchy kSZ power as a function of three parameters describing EoR: the mean free path of ionizing photons $R_{M F P}$, the ionizing efficiency $\eta$, and the minimum virial temperature $T_{v i r}$ of halos contributing to reionization. Detailed measurements of the kSZ power spectrum will place constraints on the astrophysical processes reionizing the Universe. Figure 6 in Mesinger et al. (2012); used with permission.

surements. Fisher matrix forecasts predict that adding HERSCHEL data to the SPTSZ data would improve current constraints by a factor of four.

In the medium term $(\sim 2020)$, the next generation of experiments on the ACT and SPT telescopes should lead to a substantial improvement in the kSZ constraints. These experiments will have dramatically lower noise levels and better frequency coverage, which will break the degeneracy between the two SZ effects in current data. Forecasts for these experiments are likely to be less sensitive to the CIB modeling than the HERSCHEL predictions since the data is coming from a comparatively narrow frequency range near the peak of the CMB black body. Uncertainties from SPT-3G and AdvACT are likely to be comparable, with the SPT-3G survey forecast to precisely measure the $\mathrm{kSZ}$ power with $\sigma(k S Z)=0.15 \mu \mathrm{K}^{2}$.

In the longer term (mid-2020s), results from the first large-area surveys with the planned CCAT telescope should be published. Forecasts for the proposed LWCAM instrument on CCAT are shown in Figure 4 for six frequency bands from 95 to $400 \mathrm{GHz}$. CCAT could measure the kSZ power at multiple angular scales and test the angular dependence of the kSZ power spectrum. The shape of the power spectrum encodes information on the sources and sinks of ionizing photons. Work by Mesinger et al. (2012) has shown, for instance, that slope of the kSZ power spectrum around $\ell=3000$ can inform us about the number density of Lyman Limit systems at high redshift. CCAT would also improve the measurement of the overall amplitude by another factor of two. 


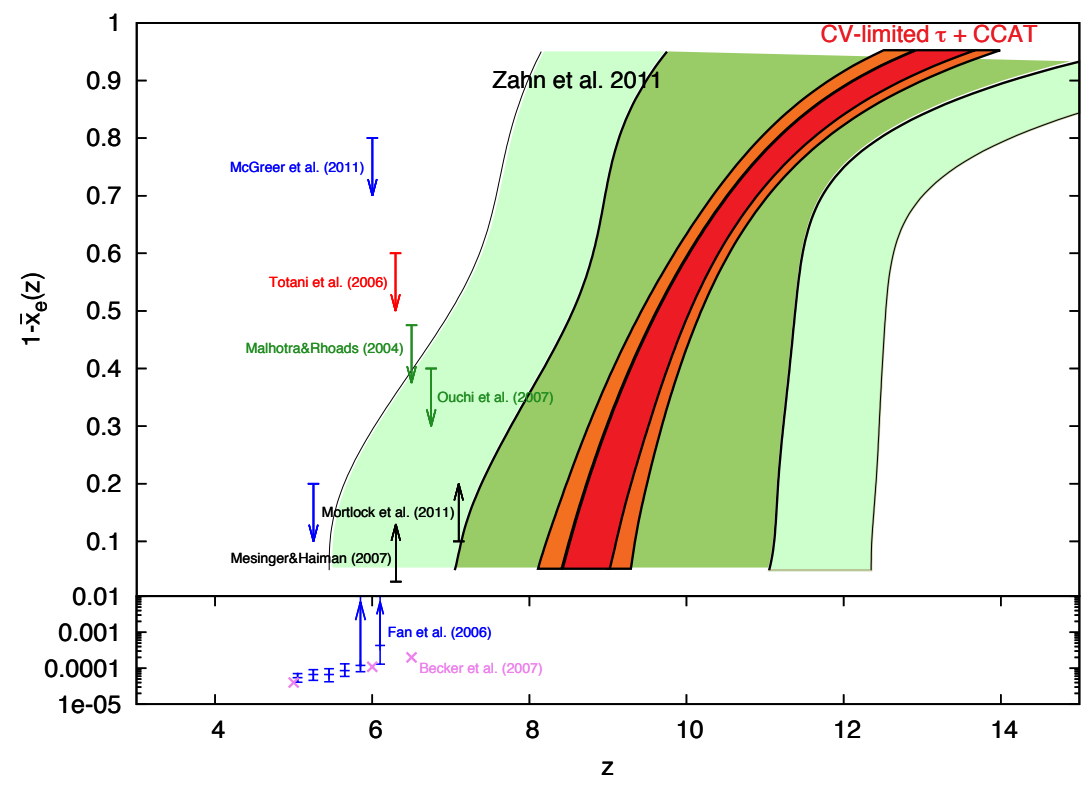

Fig. 4 CMB-derived constraints on the redshift evolution of the mean neutral fraction. The SPTSZ+WMAP 68/95\% confidence ranges for the conservative case in Zahn et al. (2012) are indicated by the dark/light green shading. Forecast constraints from CCAT plus a future cosmic variance limited measurement of the optical depth are shown by the red/orange shading. A sampling of other constraints on the neutral fraction based on quasar spectra (blue and violet constraints as well as black lower limits), a gamma ray burst (red upper limit), and Ly $\alpha$ emitters (green upper limits) is also shown. Image Credit: Oliver Zahn

\section{Other Observables}

We finally turn to two other potentially observable signatures of cosmic reionization in the CMB. First, the ionized gas will distort the CMB black-body spectrum, with the magnitude of the spectral distortion depending on the gas temperature. Second, variations in optical depth across the sky will introduce a non-Gaussian signal in the CMB temperature and polarization anisotropies. These signals have yet to be detected.

\subsubsection{Spectral distortions}

In Section 2, we described the dominant effects of Thomson scattering between the $\mathrm{CMB}$ and free electrons during the EoR: damping of anisotropy and the polarized reionization bump. Beyond these effects, the photon-electron temperature difference very slightly distorts the black body spectrum of the scattered CMB photons. This Compton y-distortion has a spectral dependence that can be parameterized as $\Delta I_{v}=$ 
$y Y_{S Z}(v)$ Zel'dovich \& Sunyaev (1969); y-distortions are commonly encountered in galaxy clusters with the thermal Sunyaev-Zel'dovich effect Sunyaev \& Zel'dovich (1972). The amplitude y can be expressed as:

$$
y=\int \frac{k\left[T_{e}(z)-T_{C M B}(z)\right]}{m_{e} c^{2}} n_{e}(z) \sigma_{T} c d z,
$$

where $\mathrm{k}$ is Boltzmann constant, $\mathrm{c}$ is the speed of light, $\sigma_{T}$ is the Thomson crosssection, the quantities subscripted by $e$ are the electron temperature, mass and number density, and $T_{C M B}$ is the CMB temperature. The magnitude scales with the optical depth, $\tau$, and electron temperature, $T_{e}$, as Kogut et al. (2011):

$$
y \simeq 1.8 \times 10^{-7}\left(\frac{\tau}{0.09}\right)\left(\frac{T_{e}}{10^{4} \mathrm{~K}}\right) .
$$

This amplitude is two orders of magnitude below the best current limit of $y<1.5 \times 10^{-5}$ at $95 \%$ confidence from the FIRAS experiment Fixsen et al. (1996). However satellite experiments have been proposed recently to improve the FIRAS measurement 10,000-fold (e.g., PIXIE Kogut et al. (2011), PRISM André et al. (2014)). For instance, PIXIE Kogut et al. (2011) is designed to achieve $\sigma_{y} \sim 10^{-9}$, and thus would present the intriguing possibility of measuring the electron temperature during EoR at the 5\% level ${ }^{5}$ The gas temperature during EoR is a function of the spectrum of the ionizing sources; measuring this temperature could distinguish between, e.g., Pop II stars and black holes Kogut et al. (2011).

\subsubsection{Variations in optical depth}

The inhomogeneity of cosmic reionization means that there will be small variations in the optical depth along different line of sights. These variations imprint a non-Gaussian signal on the temperature and polarization anisotropies, in particular correlating the polarized $\mathrm{E}$ and $\mathrm{B}$-modes. The reionization signal peaks on large scales $(\ell \sim 400)$. Properly designed estimators can leverage these correlations to reconstruct the optical depth as a function of position on the sky Dvorkin \& Smith (2009); Natarajan et al. (2013). The estimator is closely related, both in concept and form, to the well-known CMB lensing estimators (e.g., Seljak \& Zaldarriaga. 1999, $\mathrm{Hu}, 2001)$. Dvorkin \& Smith (2009) estimate that a future CMB polarization satellite, in addition to mapping the lensing B-modes, might measure the duration of reionization and mean bubble size at the $10 \%$ level. Such a measurement could tell us about how quickly reionization occurred and what kind of sources were responsible for reionization.

The main observational challenge is the faintness of the signal. Compared to the recently detected lensing B-modes Hanson et al. (2013); The Polarbear Collabo-

5 Note that there would be a degeneracy between the y-distortion induced by EoR and certain classes of alternative models that inject energy into the early Universe Chluba \& Jeong (2014). 
ration: P. A. R. Ade et al. (2014), the B-mode power induced by the variations in optical depth is lower by a factor of 10-100 depending on angular scale (see Figure 3 in Dvorkin \& Smith (2009)). Still given the high signal-to-noise $(>100 \sigma)$ expected for lensing B-modes from CMB experiments under construction (e.g., AdvACT, SPT-3G Benson et al. (2014), Simons Array), and the substantially better mapping speeds that would be achieved by the proposed stage IV ground-based experiments or the next satellite experiment, the reionization signal should eventually be detectable at high signal-to-noise. While unlikely to be competitive with $21 \mathrm{~cm}$ surveys on this timescale, mapping the variations in optical depth across the sky with the $\mathrm{CMB}$ would be an independent test of reionization scenarios.

\section{Conclusions}

The CMB has yielded two major clues into cosmic reionization to date: the optical depth and kSZ power. In this work, we have discussed the theory and observations for the two observables, as well as mentioning the possibility of constraining the temperature of the intergalactic medium during reionization and mapping variations in the optical depth across the sky.

First and most robustly, measurements of large-scale CMB polarization anisotropy can be used to determine the optical depth of the Universe due to Thomson scattering. The WMAP 9-year results favor $\tau=0.089 \pm 0.014$ Bennett et al. (2013). The optical depth due to Thomson scattering depends on the column depth of free electrons, and is roughly proportional to the redshift at which the Universe is $50 \%$ ionized. The WMAP measurement suggests the midpoint of reionization is around $z=10.6 \pm 1.1$. In the near future, the Planck satellite is expected to publish an independent measurement of the optical depth with a factor of three reduction in errors.

Second, multifrequency measurements of small-scale (few arcminutes) CMB temperature anisotropy have begun to set interesting upper limits on the $\mathrm{kSZ}$ power. When combined with simulations of the homogenous kSZ power from the fully ionized Universe, the upper limits on the total $\mathrm{kSZ}$ power suggest reionization was fairly rapid. George et al. (2015) find a 95\% CL upper limit on redshift interval in which the Universe transitioned from $20 \%$ to $99 \%$ ionized to be $\Delta z<5.4$. Measurements of the kSZ power should be substantially improved by experiments under construction or planned, with first light instruments on CCAT expected to improve upon current measurements by a factor of approximately 16 .

CMB observations have been among the first direct observational probes of the EoR, and will continue to provide new and independent tests of how cosmic reionization occurred going forward. The CMB is complementary to planned $21 \mathrm{~cm}$ experiments as the $\mathrm{CMB}$ observables probe the ionized rather then neutral gas. The main insights from the CMB so far have been on the timing of reionization, with the optical depth setting when cosmic reionization occurred and the limits on kSZ power constraining the duration of reionization. In the future, measurements of the CMB may also test of the nature of the first objects, with the shape of the kSZ 
power spectrum and variations in optical depth across the sky probing bubble sizes and spectral distortions probing the temperature of the intergalactic medium.

Acknowledgements We thank Oliver Zahn for useful discussions and for creating Figure 4 We are grateful to Elizabeth George, Kyle Story, and Andrei Mesinger for valuable feedback. We acknowledge the use of the Legacy Archive for Microwave Background Data Analysis (LAMBDA). Support for LAMBDA is provided by the NASA Office of Space Science.

\section{References}

Abazajian, K. N., et al. 2014, Astroparticle Physics, Addison, G. E., Dunkley, J., \& Bond, J. R. 2013, MNRAS, 436, 1896

André, P., et al. 2014, JCAP, 2, 6

Battaglia, N., Natarajan, A., Trac, H., Cen, R., \& Loeb, A. 2013, ApJ, 776, 83

Baumann, D., et al. 2009, in American Institute of Physics Conference Series, Vol. 1141, American Institute of Physics Conference Series, ed. S. Dodelson, D. Baumann, A. Cooray, J. Dunkley, A. Fraisse, M. G. Jackson, A. Kogut, L. Krauss, M. Zaldarriaga, \& K. Smith, 10-120

Bennett, C. L., et al. 2013, ApJS, 208, 20

Benson, B. A., et al. 2014, in Society of Photo-Optical Instrumentation Engineers (SPIE) Conference Series, Vol. 9153, Society of Photo-Optical Instrumentation Engineers (SPIE) Conference Series

Birkinshaw, M. 1999, Physics Reports, 310, 97

Calabrese, E., et al. 2014, JCAP, 8, 10

Carlstrom, J. E., Holder, G. P., \& Reese, E. D. 2002, ARAA, 40, 643

Caruana, J., Bunker, A. J., Wilkins, S. M., Stanway, E. R., Lorenzoni, S., Jarvis, M. J., \& Ebert, H. 2014, MNRAS, 443, 2831

Chluba, J., \& Jeong, D. 2014, MNRAS, 438, 2065

Clément, B., et al. 2012, A\&A, 538, A66

Crawford, T. M., et al. 2014, ApJ, 784, 143

Dunkley, J., et al. 2013, JCAP, 7, 25

Dvorkin, C., \& Smith, K. M. 2009, PRD, 79, 043003

Finkbeiner, D. P., Davis, M., \& Schlegel, D. J. 1999, ApJ, 524, 867

Fixsen, D. J., et al. 1996, ApJ, 470, 63

George, E. M., et al. 2015, ApJ, 799, 177

Gruzinov, A., \& Hu, W. 1998, ApJ, 508, 435

Hanson, D., et al. 2013, Physical Review Letters, 111, 141301

Hazumi, M., et al. 2012, in , 844219-844219-9

Howlett, C., Lewis, A., Hall, A., \& Challinor, A. 2012, JCAP, 4, 27

Hu, W. 2001, ApJ, 557, L79

Iliev, I. T., Mellema, G., Pen, U.-L., Merz, H., Shapiro, P. R., \& Alvarez, M. A. 2006, MNRAS, 369, 1625

Knox, L., Scoccimarro, R., \& Dodelson, S. 1998, Physical Review Letters, 81, 2004 
Kogut, A., et al. 2003, ApJS, 148, 161

-. 2011, JCAP, 7, 25

Lewis, A., Challinor, A., \& Lasenby, A. 2000, Astrophys. J., 538, 473

McQuinn, M., Furlanetto, S. R., Hernquist, L., Zahn, O., \& Zaldarriaga, M. 2005, ApJ, 630, 643

Mesinger, A., Ferrara, A., \& Spiegel, D. S. 2013, MNRAS, 431, 621

Mesinger, A., McQuinn, M., \& Spergel, D. N. 2012, MNRAS, 422, 1403

Mortlock, D. J., et al. 2011, Nature, 474, 616

Natarajan, A., Battaglia, N., Trac, H., Pen, U.-L., \& Loeb, A. 2013, ApJ, 776, 82

Ouchi, M., et al. 2010, ApJ, 723, 869

Page, L., et al. 2007, ApJS, 170, 335

Park, H., Shapiro, P. R., Komatsu, E., Iliev, I. T., Ahn, K., \& Mellema, G. 2013, ApJ, 769, 93

Phillips, P. R. 1995, ApJ, 455, 419

Planck Collaboration et al. 2014a, A\&A, 571, A15

-. 2014b, A\&A, 571, A16

—. 2015, ArXiv: 1502.01589

Rees, M. J. 1968, ApJ, 153, L1

Santos, M. G., Cooray, A., Haiman, Z., Knox, L., \& Ma, C. 2003, ApJ, 598, 756

Schroeder, J., Mesinger, A., \& Haiman, Z. 2013, MNRAS, 428, 3058

Seljak, U., \& Zaldarriaga, M. 1999, PRL, 82, 2636

Shaw, L. D., Rudd, D. H., \& Nagai, D. 2012, ApJ, 756, 15

Sievers, J. L., et al. 2013, JCAP, 10, 60

Smoot, G. F., et al. 1992, ApJ, 396, L1

Sobacchi, E., \& Mesinger, A. 2014, MNRAS, 440, 1662

Sunyaev, R. A., \& Zel'dovich, Y. B. 1972, Comments on Astrophysics and Space Physics, 4, 173

Tajima, O., Choi, J., Hazumi, M., Ishitsuka, H., Kawai, M., \& Yoshida, M. 2012, in , 84521M-84521M-9

The Planck Collaboration. 2006, ArXiv:astro-ph/0604069

The Polarbear Collaboration: P. A. R. Ade et al. 2014, ApJ, 794, 171

Trac, H., Bode, P., \& Ostriker, J. P. 2011, ApJ, 727, 94

Treu, T., Schmidt, K. B., Trenti, M., Bradley, L. D., \& Stiavelli, M. 2013, ApJ, 775, L29

Zahn, O., Zaldarriaga, M., Hernquist, L., \& McQuinn, M. 2005, ApJ, 630, 657

Zahn, O., et al. 2012, ApJ, 756, 65

Zel'dovich, Y. B., \& Sunyaev, R. A. 1969, Ap\&SS, 4, 301 